\title{
Time resolved structure analysis of vibrating gallium phosphate under alternating electric field
}

\author{
S. Aoyagi ${ }^{1}$, K. Miwa1 ${ }^{1}$, H. Osawa ${ }^{2}$, K. Sugimoto ${ }^{2,3}$, H. Takeda ${ }^{4}$ \\ ${ }^{1}$ Department of Information and Basic Science, Nagoya City University, Nagoya 467-8501, Japan, \\ ${ }^{2}$ Research and Utilization Division, Japan Synchrotron Radiation Research Institute, Sayo, Hyogo 679-5198, Japan, \\ ${ }^{3}$ Institute for Integrated Cell-Material Sciences (iCeMS), Kyoto University, Yoshida-Ushinomiya-cho, \\ Sakyo-ku, Kyoto 606-8501, Japan, \\ ${ }^{4}$ Graduate School of Science and Engineering, Saitama University, 255 Shimo-Okubo, Sakura-ku, Saitama, 338-8570, Japan \\ aoyagi@nsc.nagoya-cu.ac.jp
}

Piezoelectric crystals, which exhibit electric polarization under mechanical stress and a mechanical strain under an electric field, are widely used in various electro-mechanical devices such as oscillators, sensors, and actuators. The mechanism of piezoelectricity can be simply explained by displacements of cations and anions against each other under a mechanical stress or an electric field. The actual relationship between a lattice strain and atomic displacements induced by the application of an electric field should be revealed by X-ray diffraction (XRD) structural analysis under an electric field. However, atomic displacements induced by inverse piezoelectric effects are usually very small to detect by conventional XRD measurements.

We have recently succeeded in detecting such small atomic displacements in piezoelectric oscillators of $\alpha$-quartz $\left(\mathrm{SiO}_{2}\right)$ and langasitetype crystals $\left(\mathrm{La}_{3} \mathrm{Ga}_{5} \mathrm{SiO}_{14}\right.$ and $\left.\mathrm{Nd}_{3} \mathrm{Ga}_{5} \mathrm{SiO}_{14}\right)$ under alternating electric fields by using a combination of resonant mechanical vibration and time-resolved XRD [1-3]. The amplitudes of the mechanical vibration of piezoelectric oscillators under an alternating electric field were resonantly enhanced at the fundamental resonant frequency. The time dependences of the enhanced lattice strain and atomic displacements during resonant mechanical vibration were measured by time-resolved XRD using short-pulse X-rays from a synchrotron radiation source and a high-repetition-rate X-ray chopper [4]. The time-resolved crystal structure analyses of the $\alpha$-quarts and langasite-type crystals revealed that bridging angles of oxygen tetrahedra $\left(\mathrm{SiO}_{4}\right.$ and $\left.\mathrm{GaO}_{4}\right)$ are deformed with displacements of specific oxygen atoms along the applied electric field during the resonant vibrations. Deformations of specific oxygen tetrahedra were also observed in the langasite-type crystals. This seems the reason why the piezoelectric constants $d_{11}$ of the langasite-type crystals are larger than that of $\alpha$-quarts. Gallium phosphate $\left(\mathrm{GaPO}_{4}\right)$ is a piezoelectric crystal with the structure consisting of $\mathrm{GaO}_{4}$ and $\mathrm{PO}_{4}$ tetrahedra. To reveal the difference between $\mathrm{GaO}_{4}$ and $\mathrm{PO}_{4}$ tetrahedra in response to an electric field, time-resolved crystal structure analysis of a resonantly vibrating $\mathrm{GaPO}_{4}$ crystal under an alternating electric field was performed in this study.

A commercial $\mathrm{GaPO}_{4}$ oscillator with the fundamental resonant frequency of $5.8 \mathrm{MHz}$ was used in the XRD measurement under an alternating electric field. The XRD measurement was performed at beamline BL02B1 of SPring-8 large synchrotron radiation facility. Resonant vibration of the $\mathrm{GaPO}_{4}$ oscillator under a sine-wave electric field was synchronized with repetitive short-pulse X-rays with a pulse width of $50 \mathrm{ps}$ extracted at $2.6 \mathrm{kHz}$ by the high-repetition-rate X-ray chopper [4]. The time dependence of momentary XRD data was measured by tuning the delay time of the sine-wave electric field to the short-pulse X-rays. The $\beta$ and $\gamma$ angles of the $C$-centred orthorhombic lattice converted from the trigonal lattice are deformed from $90^{\circ}$ by a thickness-shear strain under an electric field. The time dependence of $\Delta \gamma=\gamma-90^{\circ}$ of the resonantly vibrating $\mathrm{GaPO}_{4}$ oscillator under an alternating electric field with the amplitude of $0.17 \mathrm{MV} / \mathrm{m}$ reaches $0.23^{\circ}$ at the maximum. The maximum $|\Delta \gamma|$ is $\sim 10^{3}$ times larger than $|\Delta \gamma|$ under a static electric field with the strength of $0.17 \mathrm{MV} / \mathrm{m}$. We revealed the differences between the crystal structures of the resonantly vibrating $\mathrm{GaPO}_{4}$ at the moments when the $\gamma$ angle reaches the minimum and maximum.

[1] Aoyagi, S., Osawa, H., Sugimoto, K., Fujiwara, A., Takeda, S., Moriyoshi, C., \& Kuroiwa, Y. (2015). Appl. Phys. Lett. 107, 201905.

[2] Aoyagi, S., Osawa, H., Sugimoto, K., Takeda, S., Moriyoshi, C., \& Kuroiwa, Y. (2016). Jpn. J. Appl. Phys. 55, 10 TC05.

[3] Aoyagi, S., Osawa, H., Sugimoto, K., Nakahira, Y., Moriyoshi, C., Kuroiwa, Y., Takeda, H., \& Tsurumi, T. (2018). Jpn. J. Appl. Phys. 57, 11UB06.

[4] Osawa, H., Kudo, T., \& Kimura, S. (2017). Jpn. J. Appl. Phys. 56, 048001.

\section{Keywords: piezoelectricity; $\mathrm{GaPO}_{4}$; time resolved structure analysis}

This work was supported by a Grant-in-Aid for Scientific Research from the Japan Society for the Promotion of Science (JSPS) (Grant No. JP19H02797) and Tatematsu Foundation. The synchrotron radiation experiments were performed at SPring-8 with the approval of the Japan Synchrotron Radiation Research Institute (JASRI) (Proposal Nos.2018B1529 and 2019A1737). 